\section{Polarization effect on the depth resolution of optical coherence tomography}

\author{
Shuliang Jiao* and Marco Ruggeri \\ University of Miami Miller School of Medicine, Bascom \\ Palmer Eye Institute, 1638 NW 10th Ave., Miami, \\ Florida 33136
}

\begin{abstract}
We find for the first time that polarization mismatch of the sample and reference arms in optical-fiberbased optical coherence tomography (OCT) has critical effect on its depth resolution when the light source is partially polarized. When the polarization states of the two arms are matched, the measured point spread function (PSF) is almost identical to the theoretical prediction. When their polarization states are mismatched, the PSF can be so distorted that the depth resolution is degraded to several times the theoretical value. When we polarize the source light with a polarizer, then the degree of polarization (DOP) is unity, and the depth resolution becomes independent of the polarization mismatch. This discovery has fundamental importance for high-resolution OCT imaging of biological tissues. With DOP $<1$, the depth resolution can be quickly degraded by either birefringence or scattering in the sample. Adjusting polarization controllers can only improve the depth resolution at a certain depth in a sample if the polarization state of light changes along the depth. When $\mathrm{DOP}=1$, uniform resolution along the depth of a sample can be achieved. () 2008 Society of PhotoOptical Instrumentation Engineers. [DOI: 10.1117/1.3037341]
\end{abstract}

Keywords: imaging; polarization; resolution; coherence; interferometers.

Paper 08245LR received Jul. 17, 2008; revised manuscript received Oct. 12, 2008; accepted for publication Oct. 14, 2008; published online Dec. 11, 2008.

Optical coherence tomography (OCT) is an interferometerbased optical imaging modality that can reveal the microscopic structures of biological samples. Both time domain (TD) and spectral domain (SD) detection techniques have been applied in OCT with great success. As in all imaging modalities, resolution is an important parameter that describes the spatial resolving capability of a system. Ultrahigh resolution OCT has been demonstrated in both TD and SD systems. By using either superluminescent diode (SLD) or femtosecond laser-based light sources, depth resolution better than $3 \mu \mathrm{m}$ in the tissue has been achieved. ${ }^{1-3} \mathrm{We}$ all know that the depth resolution of an OCT system depends on the bandwidth and the center wavelength of the light source if the spectra and dispersion of the reference and sample arms in the interferometer are well balanced., ${ }^{2,5}$ In biological tissues,

*Address all correspondence to: Shuliang Jiao, PhD, Bascom Palmer Eye Institute, University of Miami School of Medicine, 1638 NW 10th Ave., Mcknight Room 206, Miami, FL 33136. Tel: 305-4824541; Fax: 305-4824543 E-mail: sjiao@med.miami.edu scattering and birefringence can modify the polarization states of the incident sample light ${ }^{6-8}$ in addition to the polarization modification by the single mode optical fibers in the sample and reference arms. Polarization controllers are usually used in a fiber-based OCT to optimize an OCT image by changing the amplitude and orientation of birefringence in the sample or reference fiber. However, the effect of polarization mismatch on the resolution of a fiber-based OCT has not been reported.

The configuration of the OCT system is the same as in our previous publications, ${ }^{9}$ except that we did not use an optical scanner in the sample arm for the experiments. A threemodule SLD (Broadlighter, T840-HP, Superlumdiodes Limited, Moscow, Russia) with a center wavelength of $840 \mathrm{~nm}$ and a full width at half maximum (FWHM) bandwidth of $100 \mathrm{~nm}$ was used. The light first passed through a fiber-based isolator (IO-F-850-FC/APC2, OFR) and was coupled into a fiber-based Michelson interferometer. In the reference arm, a lens was used to focus the light onto a mirror. In the sample arm, an identical lens was used to focus the light either on a mirror or a sample. Polarization controllers were used in both arms to modify the corresponding light polarization states.

To test the polarization effect on the depth resolution, a mirror was first placed in the sample arm. When we adjusted the polarization controller to vary the polarization state of the reference or the sample arm, we found that the spectral interference pattern changed significantly. Figure 1(a) shows the acquired raw spectral interference pattern when the polarization states of the two arms were matched. Figure 1(b) shows the acquired raw interference pattern when the polarization states of the two arms were significantly mismatched. The normalized PSF for the two cases was calculated and shown in Fig. 1(c). When the polarization states of the two arms are matched, the PSF has a main single peak with a FWHM width of $3.8 \mu \mathrm{m}$ (the depth resolution of OCT in air). In the case of Fig. 1(b), the PSF has multiple peaks with comparable amplitudes and the calculated FWHM width is around $19 \mu \mathrm{m}$, which is a $400 \%$ increase over the best case [Fig. 1(a)]. We also measured the PSF for ten different polarization matching conditions by adjusting the polarization controller randomly. The calculated depth resolutions are shown in Fig. 1(d). For each PSF, if the amplitudes of the side lobes are lower than half of that of the main peak, only the width of the main peak was counted. Otherwise, multiple peaks were included. We found that the worse the polarization mismatch is, the more the PSF is distorted with increasing amplitude of the side lobes.

To test the dependence of the resolution distortion on the bandwidth of the light source, we repeated the experiments with only one SLD by turning the other two SLDs off. In the best situation, when the polarization states of the two arms are matched, the depth resolution is $6 \mu \mathrm{m}$. In the worst situation, the measured depth resolution is $15 \mu \mathrm{m}$, which is $150 \%$ over the best situation. We can see that the broader the bandwidth of the light source is, the more sensitive the depth resolution is to the polarization mismatch of the two arms.

We hypothesize that the distortion of the PSF is caused by the polarization property of the light source. The measured degree of polarization (DOPs) of the light after passing

1083-3668/2008/13(6)/060503/3/\$25.00 @ 2008 SPIE 


\section{JBO LETTERS}

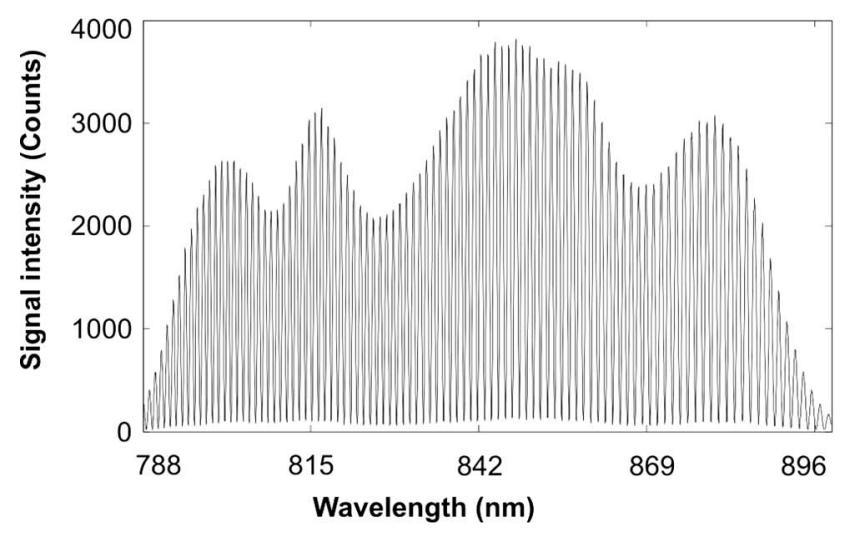

(a)

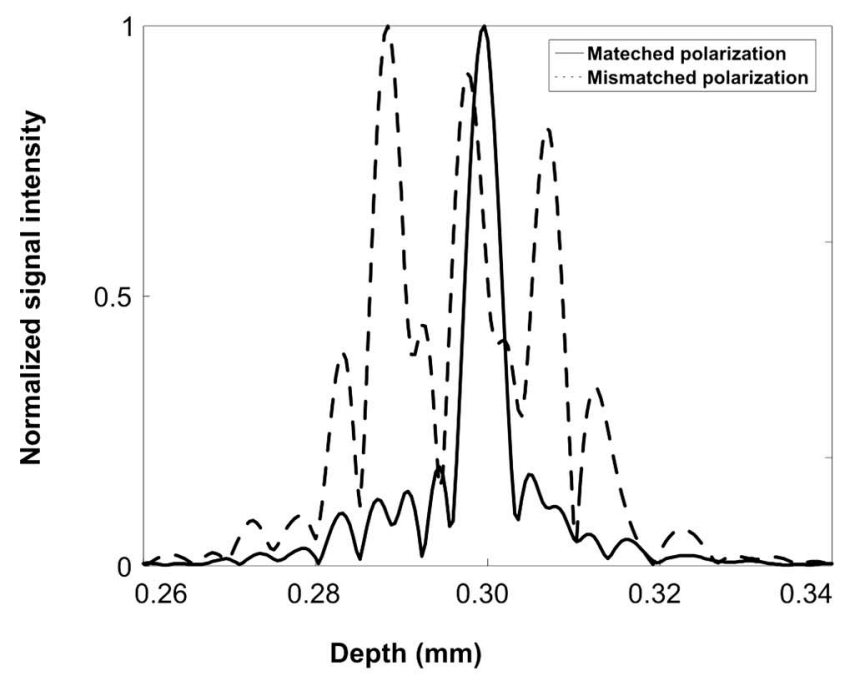

(c)

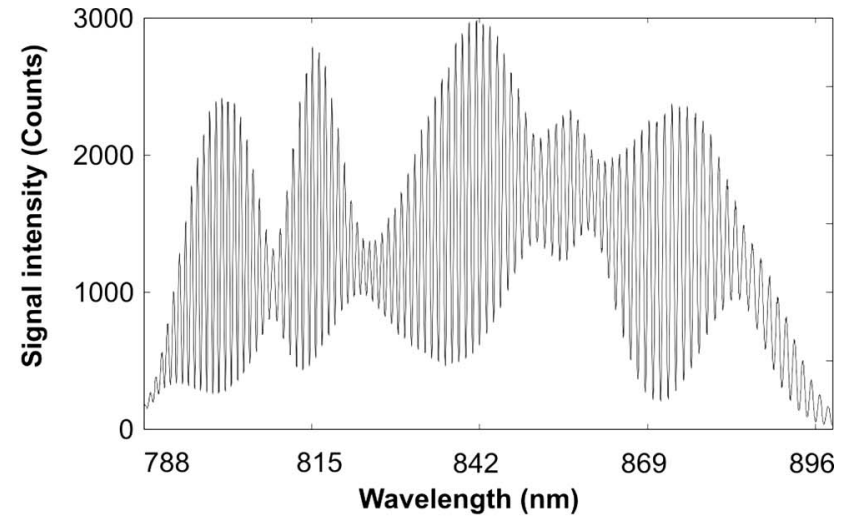

(b)

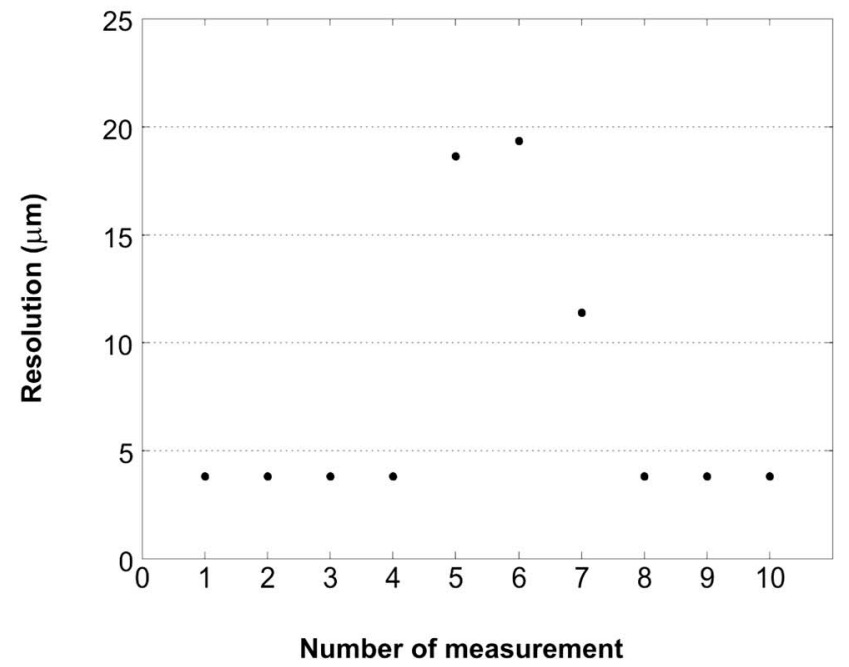

(d)

Fig. 1 Measured interference spectra and PSF for a mirror for the partially polarized three-module SLD light source: (a) the polarization states of the two arms were matched; (b) the polarization states of the two arms were significantly unmatched; (c) PSF calculated from the interference spectra in (a) and (b); and (d) depth resolution measured from ten different polarization matching conditions between the two arms.

through the isolator were 0.49 and 0.74 for the three SLDs and single SLD, respectively. So the light sources are partially polarized, in which the polarization states of light at different wavelengths are different. The polarization states of the sample and reference light at the detector at a certain wavelength can be expressed as ${ }^{8}$

$$
\begin{gathered}
\mathbf{S}_{\text {out }, s}(\lambda)=\mathbf{M}_{g} \mathbf{M}_{d} \mathbf{M}_{r s} \mathbf{M}_{s} \mathbf{S}_{\mathrm{in}}(\lambda) \quad \text { and } \\
\mathbf{S}_{\text {out }, r}(\lambda)=\mathbf{M}_{g} \mathbf{M}_{d} \mathbf{M}_{r r} \mathbf{M}_{s} \mathbf{S}_{\text {in }}(\lambda),
\end{gathered}
$$

where $\mathbf{S}_{\mathrm{in}}, \mathbf{S}_{\text {out }, s}$, and $\mathbf{S}_{\text {out }, r}$ are the incident, and output Stokes vectors of the sample and reference light at wavelength $\lambda$, respectively; $\mathbf{M}_{s}, \mathbf{M}_{r s}, \mathbf{M}_{r r}, \mathbf{M}_{d}$, and $\mathbf{M}_{g}$ are the Mueller matrix of the fiber in the source arm, the roundtrip Mueller matrix of the sample arm, the roundtrip Mueller matrix of the reference arm, and the Mueller matrices of the detection arm and the grating in the spectrometer, respectively. We can see that when we adjust the polarization controller, the matching conditions between the reference and sample polarization states vary with wavelengths. As a result, when the polarization states at certain wavelengths are matched, they may be mismatched at other wavelengths, like in the situation shown in Fig. 1(b). The nonuniform variation of the polarization matching across the wavelengths gives rise to the distortion of the PSF. From Eq. (1), we can see that if we polarize the source light, different wavelengths coming from the same arm have the same polarization state at the detector, i.e., $\mathbf{S}_{\text {out }, s}$ and $\mathbf{S}_{\text {out }, r}$ are wavelength independent. As a result, by adjusting a polarization controller, the matching conditions between the reference and sample polarization states are identical across the wavelengths, so that the depth resolution should be insensitive to polarization mismatch.

To test the hypothesis, we polarized the source light by using a linear polarizer (PCB-2.5-830, OFR) before it was coupled into the interferometer. The measured DOP $=0.9998$. The test results are shown in Fig. 2. From the test results we can see that by polarizing the source light, the depth resolution is maintained at various polarization matching conditions with only slight changes of the profiles of the side lobes. The test results proved our hypothesis.

We also tested our hypothesis by imaging a scattering and birefringent tape sample with polarized and partially polarized source light (the original SLD), respectively. The sample was placed on the focal plane of the lens in the sample arm. For 


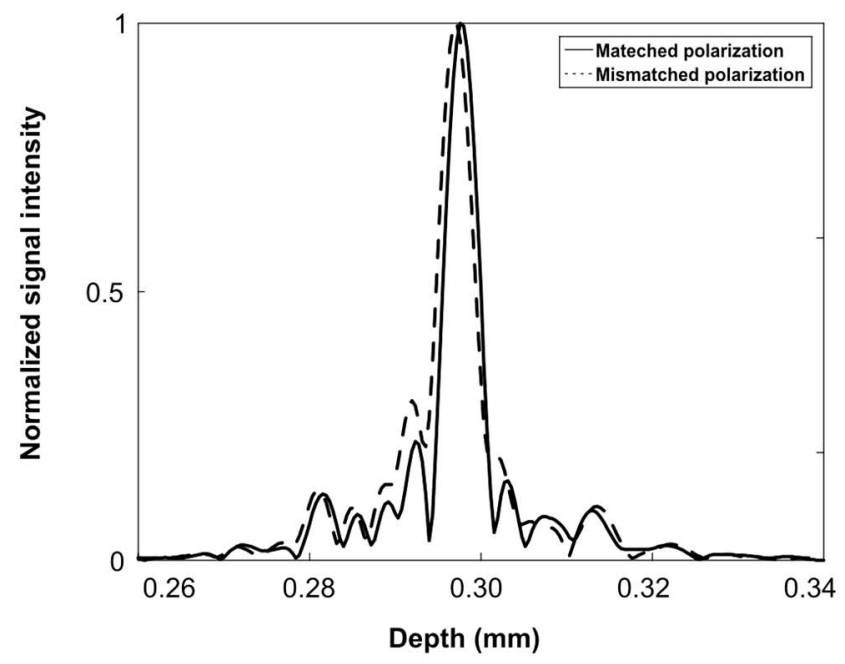

(a)

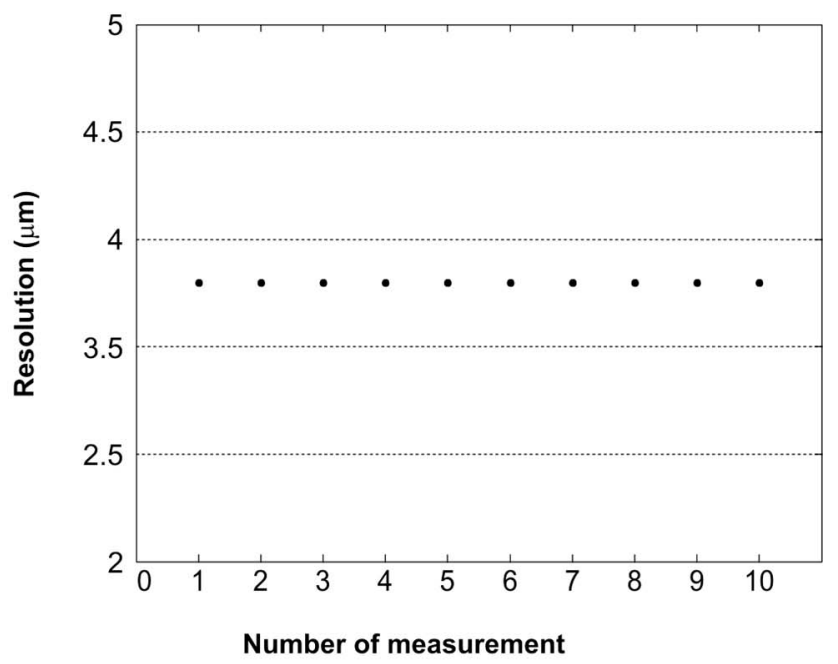

(b)

Fig. 2 Measured PSF for the light source in Fig. 1 after the light was polarized with a polarizer: (a) PSF calculated from the interference spectra and (b) depth resolution measured from ten different polarization matching conditions between the two arms.

each polarization status of the light source, we adjusted the polarization controller in the reference arm to optimize the sharpness of the peak at the front surface of the tape. We found that the shape and sharpness of the OCT signals at the front surface are similar for the partially polarized and polarized light $(\mathrm{FWHM}=3.8 \mu \mathrm{m})$. But the two signals at the back surface of the tape differed with each other significantly. In the case of partially polarized light, the resolution at the back surface was degraded significantly $(\mathrm{FWHM}=11 \mu \mathrm{m})$ with greatly increased side lobes. In contrast, for the polarized light, the shape and resolution of the OCT signal is well preserved $(\mathrm{FWHM}=4.2 \mu \mathrm{m})$. The slight widening of the OCT signal at the back surface of the tape for the polarized source light might be caused by dispersion mismatch between the two arms.

The current findings are important in OCT applications in biomedical imaging, especially for ultrahigh resolution systems. We know that scattering and tissue birefringence can modify the polarization states of the incident sample light. ${ }^{6}$ If the source light is partially polarized $(\mathrm{DOP}<1)$, modification of the sample light polarization state will seriously degrade the depth resolution, as demonstrated in the test with the tape sample. Because the modification of the polarization states varies along the depth of a sample, adjusting the polarization controller can only compensate the polarization mismatch at a certain depth of the sample with the reference arm, for example the front surface of the sample. In other words, it is impossible to have a uniform depth resolution along the sample depth for a partially polarized light source.

In conclusion, we have discovered for the first time that the polarization mismatch of sample and reference arms in singlemode optical-fiber-based OCT has critical effect on depth resolution when the light source is partially polarized. We have proved that this effect is caused by the variation of polarization states among different wavelengths of the light source. To achieve uniform resolution along the depth of a scattering or birefringence sample, the light source should be polarized.
This discovery is important for high-resolution OCT imaging of biological tissues.

\section{Acknowledgments}

This study is supported in part by the National Institutes of Health grant 1R21 EB008800-01.

\section{References}

1. W. Drexler, U. Morgner, R. K. Ghanta, F. X. Kärtner, J. S. Schuman, and J. G. Fujimoto, "Ultrahigh resolution ophthalmic optical coherence tomography," Nat. Methods 7, 502-507 (2001).

2. B. Cense, N. Nassif, T. Chen, M. Pierce, S. H. Yun, B. Park, B. Bouma, G. Tearney, and J. de Boer, "Ultrahigh-resolution high-speed retinal imaging using spectral-domain optical coherence tomography," Opt. Express 12, 2435-2447 (2004), see http:// www.opticsinfobase.org/abstract.cfm?URI=oe-12-11-2435.

3. M. Wojtkowski, V. Srinivasan, T. Ko, J. Fujimoto, A. Kowalczyk, and J. Duker, "Ultrahigh-resolution, high-speed, Fourier domain optical coherence tomography and methods for dispersion compensation," Opt. Express 12, 2404-2422 (2004), see http:// www.opticsinfobase.org/abstract.cfm?URI=oe-12-11-2404.

4. D. Huang, E. A. Swanson, C. P. Lin, J. S. Schuman, W. G. Stinson, W. Chang, M. R. Hee, T. Flotte, K. Gregory, C. A. Puliafito, and J. G. Fujimoto, "Optical coherence tomography," Science 254, 1178-1181 (1991).

5. Y. T. Pan, Z. L. Wu, Z. J. Yuan, Z. G. Wang, and C. W. Du, "Subcellular imaging of epithelium with time-lapse optical coherence tomography," J. Biomed. Opt. 12, 050504 (2007).

6. J. M. Schmitt and S. H. Xiang, "Cross-polarized backscatter in optical coherence tomography of biological tissue," Opt. Lett. 23, 10601062 (1998).

7. J. F. de Boer, T. E. Milner, M. J. C. van Gemert, and J. S. Nelson, "Two-dimensional birefringence imaging in biological tissue by polarization-sensitive optical coherence tomography," Opt. Lett. 22 934-936 (1997).

8. S. Jiao and L. H.V. Wang, "Jones-matrix imaging of biological tissues with quadruple-channel optical coherence tomography," J. Biomed. Opt. 7, 350-358 (2002).

9. H. Wehbe, M. Ruggeri, S. Jiao, G. Gregori, C. A. Puliafito, and W. Zhao, "Automatic retinal blood flow calculation using spectral domain optical coherence tomography," Opt. Express 15, 15193-15206 (2007), see http://www.opticsinfobase.org/abstract.cfm?URI=oe-1523-15193. 